\title{
The Study of a Simple Pine-Oil Based Laboratory Prepared and Commercial Detergents Using Conductivity Measurements
}

\author{
Mosotho J. George*, Ts'ukulu Motsamai \\ Department of Chemistry and Chemical Technology, National University of Lesotho, Roma, Lesotho \\ Email: "jm.george@nul.Is, maluti2005@gmail.com
}

Received 8 October 2015; accepted 16 November 2015; published 19 November 2015

Copyright (C) 2015 by authors and Scientific Research Publishing Inc.

This work is licensed under the Creative Commons Attribution International License (CC BY). http://creativecommons.org/licenses/by/4.0/

(c) (i) Open Access

\section{Abstract}

Detergents are very important substances in everyday life as they are used in laundry services. This manuscript reports the study of the commercial and laboratory prepared pine oil-based detergents employing the critical micelle concentration (CMC) phenomenon using conductivity measurements. The two samples showed the CMC values of $0.0725 \mathrm{~g} / \mathrm{cm}^{3}$ and $0.0920 \mathrm{~g} / \mathrm{cm}^{3}$ for laboratory and commercial samples respectively. The effect of ionic strength was investigated using $\mathrm{NaCl}$ and it demonstrated a drop of CMC value of about CMC by $40 \%$ (laboratory sample) and $70 \%$ (commercial sample) while the equi-molar naphthalene increased the CMC values by about $50 \%$ (laboratory sample) and $12 \%$ (commercial sample) relative to their corresponding values under distilled water. The combined effect of equi-molar NaCl and naphthalene lowered the CMC by 5\% (laboratory sample) and $30 \%$ (commercial sample). These differences could signify the superiority of the laboratory sample in that it is somehow buffered against drastic changes in the CMC under different conditions. The relationship between conductivity and the CMC values does not show sufficient linearity $\left(R^{2}<\right.$ 0.8403) suggesting different mechanisms of interactions between $\mathrm{NaCl}$ and naphthalene. Overall, the results are gratifying to the small-scale manufacturer who supplied the preparation protocol for laboratory sample preparation, in two respects: they inspire some degree of confidence in his product as well as enabling the manufacturer to employ the same protocol for his quality control practices as such improve product consistency and hence profitability.

\section{Keywords}

Detergent, Pine Gel, Critical Micelle Concentration, Conductivity, Ionic Strength, Small-Scale

\footnotetext{
${ }^{*}$ Corresponding author.
}

How to cite this paper: George, M.J. and Motsamai, T. (2015) The Study of a Simple Pine-Oil Based Laboratory Prepared and Commercial Detergents Using Conductivity Measurements. American Journal of Analytical Chemistry, 6, 957-964. 


\section{Manufacturer}

\section{Introduction}

Detergents and soaps are chemicals used in laundry to aid the removal of dirt or foreign matter from surfaces. Their formulations commonly comprise essential constituents (surface active agents) and subsidiary constituents (builders, boosters, fillers and auxiliaries) that wet the fabric, matter, solubilize grime (accumulation of dirt), keep the soil in suspension, freshen, removing odour as well as brightening the fabrics as they clean. Generally these are products of the reactions between long chain fatty acids with alkali metals. Soaps are made from fats and oils, or their long chain fatty acids, by treating them chemically with a strong alkali [1]. Their properties depend on the chain length of fatty acids in the blend, amount of unsaturation, formulation and the soap's intended applications [2] [3]. The detergents on the other hand use the sulfonated acid instead of a normal carboxylic acid moiety resulting in a slightly harsher "soap" called a detergent, which is mostly used in multi-purpose cleaning more than in bathing. Higher molecular weight detergents are claimed to give superior detergency compared to the lower molecular weight counterparts [4] [5].

Several approaches have been reported for estimating or determining the detergency of some simple detergents, namely, the hydrophilic-lipophilic balance (HLB) approach [6], the phase inversion temperature (PIT) approach [7], as well as the Critical Micelle Concentration (CMC) approaches. It was not clear from literature when CMC was firstly reported unlike the other two with the earliest report being 1971 [8]. The HLB and CMC approaches are the most commonly used approaches probably due to the fact that PIT requires that analytes are temperature stable, an attribute surfactants do not have [9]. The HLB was developed as an empirical scale where oleic acid and its salt, sodium oleate were used as standards with values of 1 and 20 respectively, and other compounds (analytes) were compared and placed accordingly on this scale. It has, however, been argued lately that the HLB approach only gives approximate values since some arbitrary scale is deployed and the values obtained in the original experiments by Griffin are not experimental but rather calculated from theoretical formulae [10].

Critical micelle concentration on other hand measures the maximum concentration that monomers of the surfactant can exist before they aggregate into micelles, vesicles, micro-emulsions, bilayers, membranes and liquid crystals [11]. Micelles are the most predominant aggregate structures in surfactant solutions and form over a narrow range of surfactant concentration-the CMC. These are spontaneously formed aggregated units with the hydrophobic ends forming the core while the hydrophilic ends form a surface hydrated by water molecules [12] [13]. Due to this behaviour, surfactants have a higher degree of ionization below the CMCthus behave as "strong electrolytes", while above the CMC, they are partially ionised due to micelles formation reducing ion mobility. This micellarisation, therefore, results in the change in most physical properties such as electrical conductivity, surface tension, osmotic pressure, density and light scattering or refractive index [14].

This change in the properties has enabled this technique to be applicable to the use of CMC approach on determining the strength of the detergency of ordinary surfactants used as detergents or soaps [15] [16]. Different approaches have been reported for determination of CMC of detergents. However, conductivity is by far the easiest, most affordable and quite versatile as demonstrated by its application in deducing the mechanism of metal uptake by solid adsorbents [17]. In principle, the increase in electrical conductance below the CMC is usually higher than after the CMC due to the presence of freely moving ions of both the monomers and the salt ions before the CMC, while after the CMC, there is less conductivity since the organic moieties are bound into the micelles, and as such they are not free to move, thus reducing conductivity [12] [18]. Though this approach (CMC) seems to perform satisfactorily, it is criticised for being too subjective since it requires visual inspection of the point at which the change in the physical property takes place.

This manuscript reports the study of the detergency of a simple laboratory prepared pine oil based detergent compared with the commercial brand using conductivity measurements. The laboratory sample was prepared following a protocol obtained from a local small-scale detergents manufacturer while a commercial brand was obtained from a local grocery store just outside of the main University campus in Roma, Lesotho. Since the chemical composition of the commercial detergent was known, the one that looks similar to the laboratory pre- 
pared was used for the comparison.

\section{Experimental}

\subsection{Materials and Methods}

The industrial grade reagents, namely, 96\% dodecyl-benzenesulphonic acid (CAS No. 25155-30-0), pine oil, sodium hydroxide and "green dye" were obtained from LEFCHEM—a local small-scale manufacturer in Lesotho. Besides these, all other reagents used were of analytical grade: methanol, naphthalene, sodium chloride and sodium hydroxide were obtained from ACE (Johannesburg South Africa). Deionized water was prepared in-house using simple water still. The commercial Pine Gel (trade name) detergent was obtained locally from a grocery store. Conductivity measurements were performed using a HANNA conductometer HI 8033 and HANNA membrane pH-meter HI 8314.

\subsection{Preparation of $200 \mathrm{~mL}$ In-House Pine Gel Detergent (the Manufacturer's Protocol)}

To a $24 \mathrm{~mL}$ of dodecyl-benzenesulphonic acid in a beaker, was added $72 \mathrm{~mL}$ of cold water, followed by $41 \mathrm{~mL}$ of $2 \mathrm{M} \mathrm{NaOH}$ added gradually while the mixture was being stirred continuously and the $\mathrm{pH}$ was checked using litmus. Once the solution was neutral, $52 \mathrm{~mL}$ of water were added and stirred appropriately. Appropriate amount of dye was added to get the appropriate visual appearance. The resulting detergent was left to cure to a semisolid gel. The appearance of the laboratory sample was compared with the commercial sample for appearance and consistency (see Figure 1).

\subsection{Characterisation of the Produced Detergent by CMC Determination}

A detergent stock solution of $5 \mathrm{mM}$ concentration was prepared by dissolving the appropriate mass in small volume of methanol and diluted with distilled water. A test solution was prepared by adding $50 \mathrm{~mL}$ of distilled water in to an Erlenmeyer flask, to which $1.0 \mathrm{~mL}$ aliquots of the detergent stock solution were added at the time and stirred continuously using a magnetic stirrer as the resultant conductivity measured. Meanwhile a plot of conductivity against the volume of the detergent solution added was being continuously carried out. This procedure was repeated until the concentration of the detergent was higher than the CMC level (after a break in the linearity—see Figure 2).

To determine the effect of different additives on the CMC, the same procedure was repeated for different fresh detergent solutions in the presence of equi-molar $\mathrm{NaCl}$, naphthalene and the naphthalene- $\mathrm{NaCl}$ mixture, respectively.

\section{Results and Discussions}

\subsection{Preparation and Modification a Laboratory Pine Gel Detergent}

Figure 1 shows the photograph of the commercial and laboratory samples of the detergent. The laboratory pro-

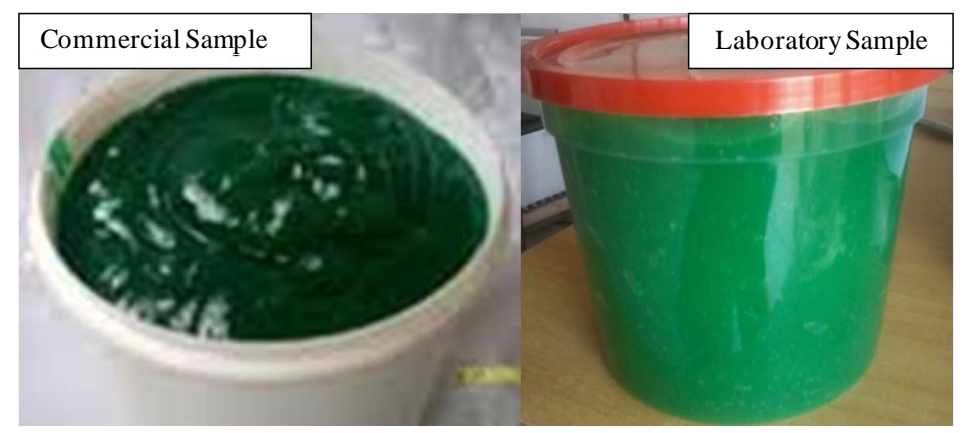

(a)

(b)

Figure 1. A picture of the detergents in the commercial packaging: (a) The laboratory prepared sample; and (b) The commercial brand. 


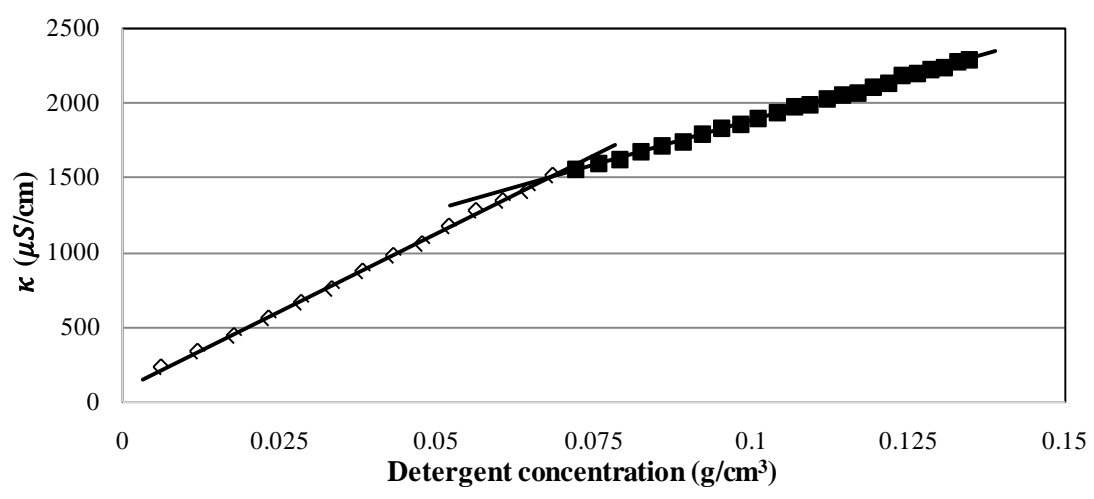

Figure 2. Variation of specific conductivity against the detergent concentration (laboratory sample).

duct matched the commercial product both in colour, texture and consistency.

As it can be seen from Figure 1, the two samples looked similar. It was not only their visual appearances, but also their feel and consistencies were comparable.

\subsection{Characterisation of the Two Detergent Samples by Critical Micelle Concentration Approach}

The critical micelle concentrations of the two detergents were determined using conductivity measurement method. The plot of conductivity versus surfactant concentration shows two straight lines with different slopes (Figure 2).

The initial part (in the range 0 - 0.07) of the plot in Figure 2, represents the conductivity of detergent surfactant before CMC (about $0.0725 \mathrm{~g} / \mathrm{cm}^{3}$ ) where only monomers of the surfactant exist in solution, while the later section (above the $0.0725 \mathrm{~g} / \mathrm{cm}^{3}$ ) shows the conductivity of the surfactant after the formation of micelles. The intersection of the two lines is taken as the CMC of the detergent as it has been discussed in the introduction.

The explanation for this behaviour is that, as a principle, at low concentrations of the surfactant, below the CMC, an ionic surfactant is completely dissociated, as such there is a linear relationship between the specific conductivity $(\kappa)$ of the surfactant solution and its concentration as the surfactant monomers behave as normal electrolyte, Kohlrausch's law is obeyed (Equation (1)). On the other hand, at concentrations above the CMC, specific conductivity is independent of surfactant concentration as the micelles behave like a weak electrolyte (partially dissociate into ions) and this is explained by (Equation (2)).

Equation 1: $\kappa=\left(\lambda_{\text {cation }}+\lambda_{\text {anion }}\right)[\text { surfactant }]^{\prime}$

Equation 2: $\kappa=\left(\lambda_{\text {cation }}+\lambda_{\text {anion }}\right) c m c+\lambda_{\text {cation }} \alpha[\text { surfactant }]^{\prime \prime}+\lambda_{\text {micelle }}[$ micelle $]$

where $\lambda_{\text {cation }}+\lambda_{\text {anion }}$, [surfactant $]^{\prime}, \lambda_{\text {micelle }},[\text { surfactant }]^{\prime \prime}$, and $\alpha$ represent conductivity of the cation, anion, concentration of surfactant below CMC, conductivity of a micelle, fractional micellar ionization, and degree of counter-ion dissociation respectively [19].

\subsection{Determination of the Effect of Electrolyte ( $\mathrm{NaCl}$ ) on the $\mathrm{CMC}$}

Figure 3 presents the plot of specific conductivity versus concentration of surfactant in the presence of $5 \mathrm{mM}$ sodium chloride to increase the ionic strength of the mixture.

As expected the CMC value dropped in the presence of $\mathrm{NaCl}$ (from 0.0725 to 0.0520 ). This trend is consistent with the available literature in that when a salt is added to a micellar solution, the CMC decreases due to the effect of the electrolyte counter-ion [20]. The main reason of lowering of CMC upon addition of an electrolyte is the "salting out" effect. Once the electrolyte is added, the ionic power of the solution increases, the CMC decreases and the transition from spherical to cylindrical shape along with increase of the micelle aggregation number occurs. The hydrophilic part of surfactant releases counter (molecular negatively charged) ions and these ions decrease the CMC value. These counter-ions create Coulombic shielding of the electrostatic repulsions among charged hydrophilic parts of ionic molecules on the micelle surface. CMC of the ionic surfactant is 


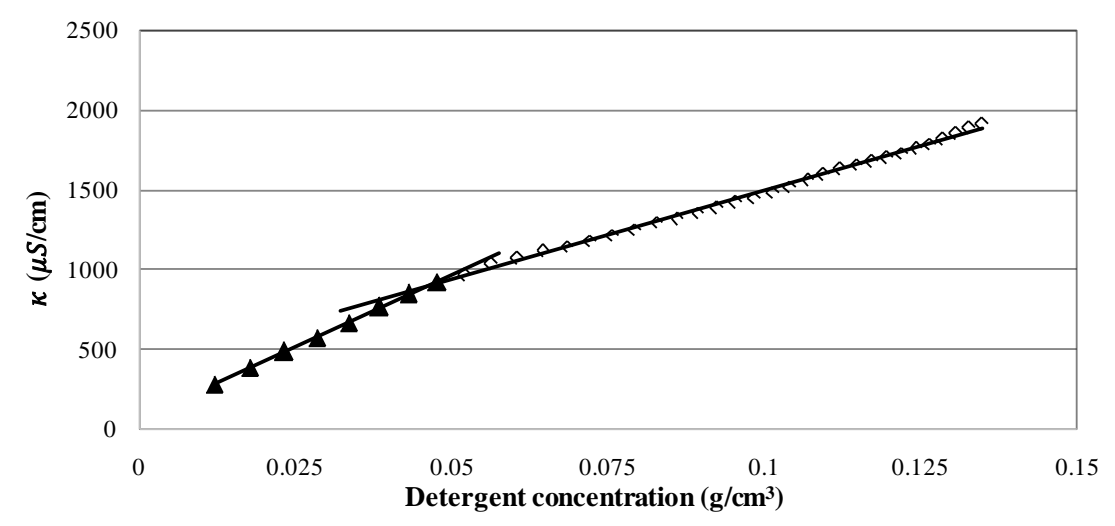

Figure 3. Variation of specific conductivity with detergent concentration in the presence of sodium chloride.

also decreased by electrolyte dehydration activity. Solutions of electrolytes remove the lyosphere of surfactants and, as a consequence, ions become strongly hydrated [21] [22].

\subsection{Effect of the Presence of an Organic Compound (Naphthalene) on the CMC}

Following the determination of the effect of the ionic strength, a fresh mixture was prepared, to which naphthalene, an organic compound with relatively high hydrophobicity, was added. Figure 4 shows the effect of naphthalene addition to the CMC of the detergent. The value of the CMC increased from 0.0725 to 0.1109 (53\% increase).

When naphthalene is added to the micellar solution, it increases the hydrophobicity of surfactant molecules. Naphthalene molecules infiltrate between the surfactant monomers' tails, increasing the radius of the micelle formed hence the increase in the CMC. A higher number of surfactant monomers together with naphthalene molecules will aggregate themselves to form larger micelle molecules. This shows that at low concentration of surfactant less number of micelle molecules are formed and most of the monomers are freely floating in the solution. As the number of monomers is increased, there is more association leading to micelle formation although much fewer in number than in the case of $\mathrm{NaCl}$ which acts in the opposite in that it draws away the water particles away from the organic surfactants thus forcing them to come together into the micelles.

\subsection{Effect of the Combination of Organic Compound (Naphthalene) and an Electrolyte $(\mathrm{NaCl})$ on the CMC}

Whereas sodium chloride decreases the CMC of detergent while the converse is true with naphthalene, when the two compounds are added together in the detergent solution, the value of CMC lies between the CMC values of the two compounds when added to the detergent solutions individually. But the value of CMC in this case is still greater than when only water is added to the detergent solution (about 35\%). This shows that naphthalene has stronger effect on CMC than sodium chloride, that is; the magnitude at which naphthalene increases CMC is greater than the magnitude at which sodium chloride decreases CMC value using water-surfactant solution as the reference point.

Figure 5 shows the four plots combined to show the CMC values for detergent solutions in different conditions, namely, in distilled water (a), in $\mathrm{NaCl}$ (b), in naphthalene (c) and in a mixture of $\mathrm{NaCl}$ and naphthalene (d).

The analytical parameters demonstrated by the plots in Figure 5 are summarised in Table 1 showing the coordinates for the CMC values.

As can be seen from the values of the slopes before the CMC compared to those higher than the CMC, clearly conductivity increases much faster at concentrations below the CMC than higher at the concentrations higher than the CMC as has been discussed prior. Despite the seeming correlation between critical micelle concentration and the conductivity values in Table 1 , this relationship is not significantly linear $\left(\mathrm{R}^{2}=0.8403\right)$ confirming the existence of different types of interactions of the detergent surfactant with naphthalene and sodium chloride. 


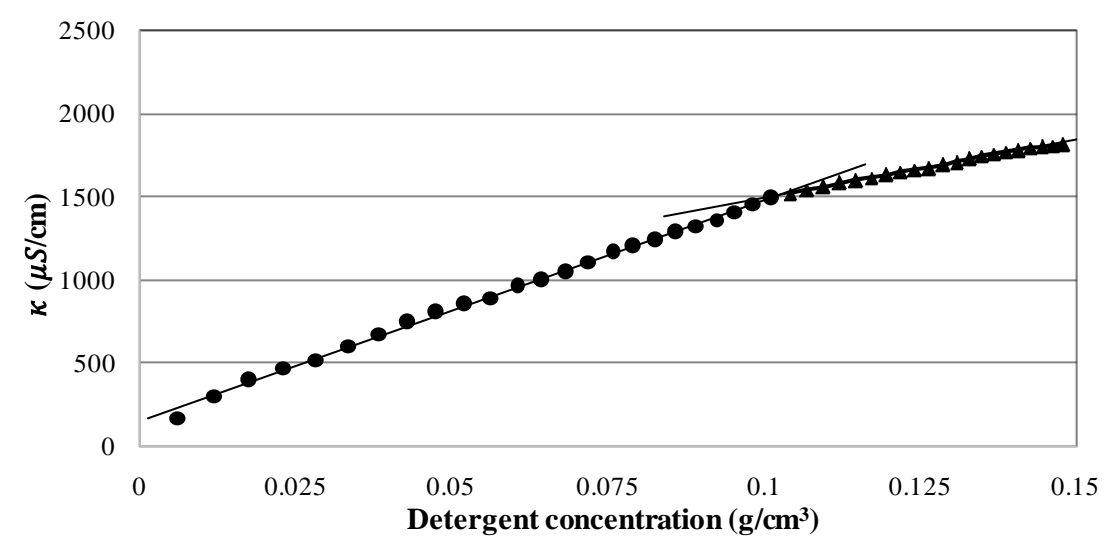

Figure 4. Variation of specific conductivity with detergent concentration after adding naphthalene.

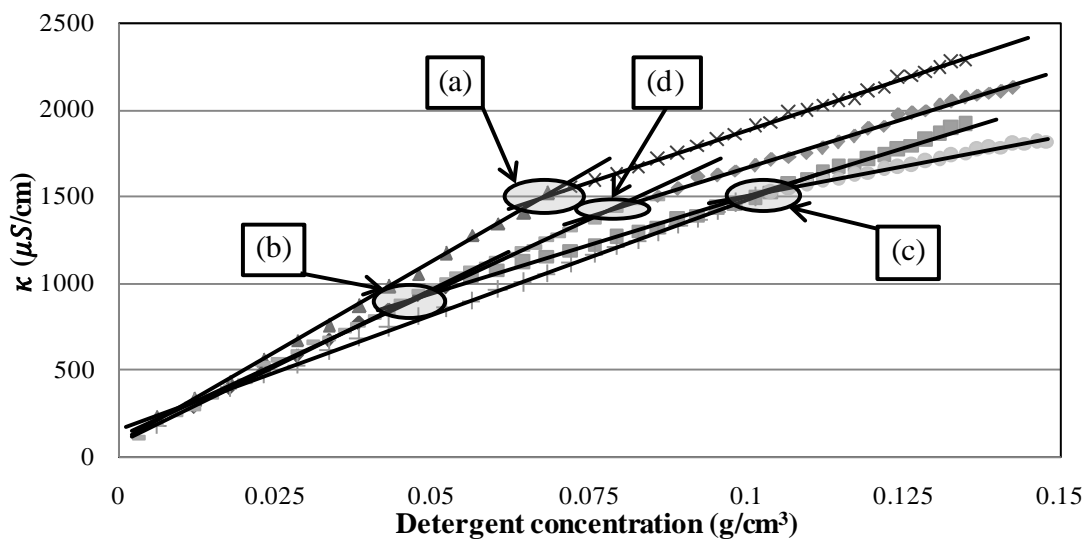

Figure 5. The comparison of the variation in specific conductivity in the presence of different additives.

Table 1. A summary of CMC values and the corresponding conductivities in different media.

\begin{tabular}{|c|c|c|c|c|c|c|}
\hline \multirow{2}{*}{ Solution composition } & \multicolumn{2}{|c|}{ Correlation coefficient, $\mathrm{R}^{2}$} & \multicolumn{2}{|c|}{ Slope } & \multirow{2}{*}{ CMC $\left(\mathrm{g} / \mathrm{cm}^{3}\right)$} & \multirow{2}{*}{$\begin{array}{c}\text { Conductivity k } \\
(\mu \mathrm{S} / \mathrm{cm})\end{array}$} \\
\hline & Before CMC & After CMC & Before CMC & After CMC & & \\
\hline Distilled water & 0.9951 & 0.9924 & 14694 & 8669 & 0.0725 & 1430 \\
\hline $\mathrm{NaCl}$ & 0.9966 & 0.9972 & 17104 & 11714 & 0.0520 & 959 \\
\hline Naphthalene & 0.9984 & 0.9926 & 12749 & 4802 & 0.1109 & 1511 \\
\hline Naphthalene + $\mathrm{NaCl}$ & 0.9952 & 0.9927 & 14694 & 8669 & 0.0693 & 1330 \\
\hline
\end{tabular}

Figure 6 shows the comparison of the two samples, laboratory prepared and the commercial sample, upon different media (in the absence and presence of $\mathrm{NaCl}$, naphthalene).

Clearly the two products do not seem to be comparable. The major difference that seems significant is the CMC value without any additives with the CMC values of $0.0725 \mathrm{~g} / \mathrm{cm}^{3}$ (laboratory sample) and $0.0920 \mathrm{~g} / \mathrm{cm}^{3}$ (commercial sample)_accounting for a difference of about 25\% relative to the laboratory sample. However, since: i) too few (3) replicate measurements were made, ii) the other parameters are comparable, iii) the linearity of the curves before and after the CMC were satisfactory (minimum $\mathrm{R}^{2}$ of 0.9915 for commercial sample after the CMC for the distilled water case), then it could be argued that this deviation was just a result of an experimental error; hence the results could be improved by taking several replicate measurements and using multiple samples as well. 


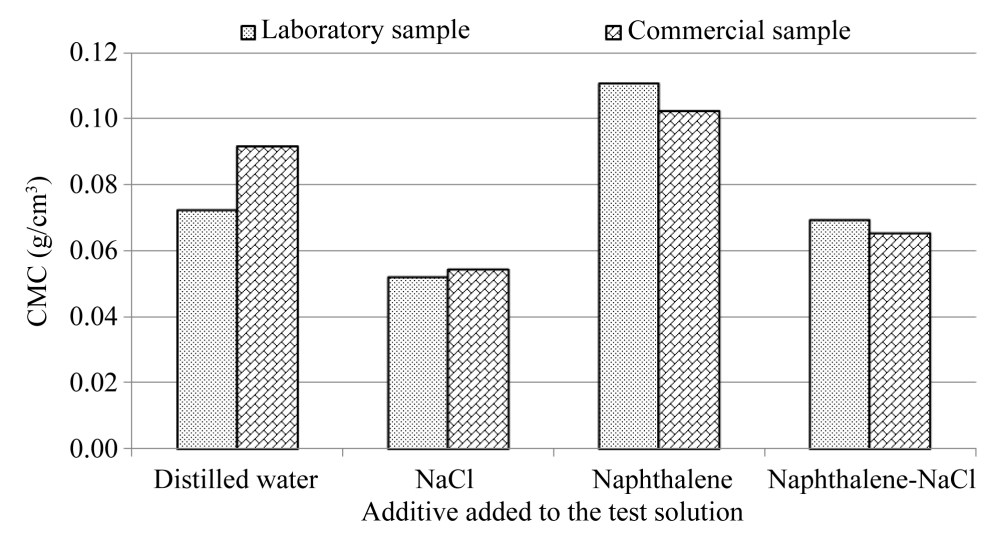

Figure 6. A comparison of the CMC values for the laboratory and commercial samples under different conditions.

\section{Conclusions}

The results presented in this project have shown that indeed critical micelle concentration approach can indeed be used for the determination of the detergency of different products. However, due to the fact that no statistics was performed, the results cannot be validated beyond presenting just a bird's eye view of the approach. Since the two formulations exhibited different magnitudes of shifts under different conditions, it could be argued that they are probably not identical although they look and feel the same. This is interesting given that the smallscale manufacturer that supplied the protocol claimed to have received it from one recognisable and experienced manufacturer in the neighbouring South Africa.

The results further demonstrated that the effect of $\mathrm{NaCl}$ decreased the $\mathrm{CMC}$ by $40 \%$ (laboratory sample) and $70 \%$ (commercial sample). Naphthalene on the other hand increased the CMC values for both samples by just over $50 \%$ (laboratory sample) and about $12 \%$ for the (commercial sample). A combination of equi-molar $\mathrm{NaCl}$ and naphthalene resulted in the decrease of the CMC by about 5\% (laboratory sample) and $30 \%$ for the commercial sample. The decrease in CMC for the combined additives indicates that the interactions of the naphthalene molecules are weaker than those from $\mathrm{NaCl}$ ions and hence the result could be the salting out of the detergent in the form of too many tiny micelles, thus could reduce the detergency of the products. While it is a good attribute to have a detergent with a lower CMC, this has to be considered with care lest the detergent loses activity completely by salting out of the washing solution.

With the application of the conductivity measurements confirmed, more work will be carried out to determine the effect of other several parameters amenable to laundry services such as effect of temperature on the CMC, with a hope of characterising the laboratory sample for the small-scale manufacturer as a way of contributing to building his business further in an effort to practice the so-called "third mission" of higher education-incubation and mentorship of small business. Certainly, conductometer is a very simple and easy gadget; hence the manufacturer can easily afford it so that they can put some CMC data on the label to improve confidence of the customers in their product.

\section{Acknowledgements}

The authors gratefully acknowledge National University of Lesotho and LEFCHEM for supporting this project.

\section{References}

[1] Scharf, W. and Malerich, C. Technocal Lecture, Natural Sciences/Chemistry, Baruch College, New York. https://www.baruch.cuny.edu/wsas/academics/natural.../chm.../9_soap.pdf

[2] National Programme on Technology Enhanced Learning (NPTEL) (n.d.) Chemical Technology (Organic) Module IV. http://nptel.ac.in/courses/103107082/module4/lecture1/lecture1.pdf

[3] Pavia, D.L., Lampaman, G.M., Kriz, G.S. and Engel, R.G. (2007) Introduction to Organic Laboratory Techniques. 4th Edition, Thomson Brooks/Cole, Washington DC, 243.

[4] Smithson, H.L. (1965) Light Duty Liquid Detergent. United States Patent No. US3175978 A. 
[5] Batchelor, N.S. (2013) Laundry Detergent Compositions Comprising Polyalkoxylated Polyethyleneimine. World Patent Application No.WO2013171210.

[6] Griffin, W.C. (1949) Classification of Surface-Active Agents by "HLB”. The Journal of the Society of Cosmetic Chemists, 1, 311-326.

[7] Shinoda, K. (1969) The Comparison between the PIT System and HLB-Value System to Emulsifier Selection. Proceedings of the 5th International Congress of Surface Activity, Barcelona, 2, 275-283.

[8] Mukerjee, P. and Mysels, K.J. (1971) National Institute of Standards and Technology: NSRDS-NBS 36. Washington DC.

[9] Orafidiya, L.O. and Oladimeji, F.A. (2002) Determination of the Required HLB Values of Some Essential Oils. International Journal of Pharmaceutics, 237, 241-249. http://dx.doi.org/10.1016/S0378-5173(02)00051-0

[10] Pasquali, R.C., Taurozzi, M.P. and Bregni, C. (2008) Some Considerations about the Hydrophilic-Lipophilic Balance System. International Journal of Pharmaceutics, 356, 44-51. http://dx.doi.org/10.1016/j.ijpharm.2007.12.034

[11] Khan, A.S. and Shah, S.S. (2008) Determination of Critical Micelle Concentration (Cmc) of Sodium Dodecyl Sulfate. (SDS) and the Effect of Low Concentration of Pyrene on Its Cmc Using ORIGIN Software. Journal of Chemical Society of Pakistan, 30, 186-191.

[12] Dominguez, A., Fernandez, A., Gonzalez, N., Iglesias, E. and Montenego, L. (1997) Determination of Critical Micelle Concentration of Some Surfactants by Three Techniques. Journal of Chemical Education, 74, 1227-1231. http://dx.doi.org/10.1021/ed074p1227

[13] Dongherty, E.V.A. and Dennis, A. (2006) Physical Organic Chemistry. University Science Books, Sausalito.

[14] Prazeres, T.J.V., Beija, M., Fernandes, F.V., Marcelino, P.G.A., Farinha, J.P.S. and Martinho, J.M.G. (2012) Determination of the Critical Micelle Concentration of Surfactants Block Copolymers Using Coumarin 153. Inorganica Chimica Acta, 381, 181-187. http://dx.doi.org/10.1016/j.ica.2011.09.013

[15] Lincolnwood, B.S., Buffalo Grove, I.R. and Mundelein, A.M. (1999) Heavy Duty Liquid Detergent Compositions Comprising Salts of $\alpha$-Sulfonated Fatty Acid Methyl Esters and Use of $\alpha$-Sulphonated Fatty Acid Salts to Inhibit Redeposition of Soil on Fabric. United States Patent No. 5945394.

[16] Calbiochem. EMD Biosciences. http://sevierlab.vet.cornell.edu/resources/CalbiochemDetergentsBook.pdf

[17] George, M.J. and Ramollo, N. (2014) A Study of the Dynamics of Copper(II) Ions Uptake from Aqueous Solutions by Human Hair Using Conductivity and pH Measurements. European Chemical Bulletin, 3, 883-887.

[18] Kaufmann, T.C., Engel, A. and Rémigy, H.-W. (2006) A Novel Method for Detergent Concentration Determination. Biophysical Journal, 90, 310-317. http://dx.doi.org/10.1529/biophysj.105.070193

[19] Tyowua, A.T., Yiase, S.G. and Wuanna, R.A. (2012) Manipulation of Concentration-Conductivity Data of Sodium Dodecyl Sulphate and Sodium Dodecylbenzene Sulphonate in $\mathrm{KCl}$ Solution in Relation to Micellisation Parameters. Chemical Sciences Journal, 79, 1-9.

[20] Fuguet, E., Rafols, C., Roses, M. and Bosch, E. (2005) Critical Micelle Concentration of Aqueous Buffered and Unbuffered Systems. Analytica Chimica Acta, 548, 95-100. http://dx.doi.org/10.1016/j.aca.2005.05.069

[21] Strnadová, H. and Kvítek, L. (2005) Micellar Systems-Factors Influencing Critical Micelle Concentration and Measuring Methods. In: Kameníček, J. and Ševčík, J., Eds., Collected Reports of the Natural Science Faculty, Palacký University of Olomouc, AUPO Chemica, 44, 7-24.

[22] Saien, J. and Asadabadi, S. (2014) Salting out Effects on Adsorption and Micellization of Three Imidazolium-Based Ionic Liquids at Liquid-Liquid Interface. Colloids and Surfaces A: Physicochemical and Engineering Aspects, 444, 138-143. http://dx.doi.org/10.1016/j.colsurfa.2013.12.060 